\title{
O uso da plataforma Khan Academy para o ensino de matemática no Brasil: um mapeamento sistemático
}

Reury Rabynne Alves da Silva - CCHE/UEPB - reury.silva@aluno.uepb.edu.br Ana Emília Victor Barbosa Coutinho - CCHE/UEPB - anaemilia@servidor.uepb.edu.br

Resumo. A inserção de tecnologias digitais na educação permite ao professor a adoção de novas possibilidades pedagógicas que visem minimizar as dificuldades enfrentadas pelos estudantes em disciplinas como a matemática. Nesse contexto, a plataforma Khan Academy oferece aos estudantes um aprendizado mais ativo, dinâmico e personalizado, ao mesmo tempo em que permite ao professor acompanhar o desempenho de suas turmas e de cada aluno individualmente. Neste trabalho, apresentamos os resultados de um mapeamento sistemático da literatura que teve como propósito identificar os trabalhos publicados nos últimos cinco anos que propõem o uso da plataforma Khan Academy para o ensino de matemática no Brasil. Os resultados indicam que a plataforma Khan Academy vem sendo inserida aos poucos no processo de ensino e aprendizagem de matemática em todos os níveis de educação no Brasil.

Palavras-chave: ensino de matemática, plataforma Khan Academy, mapeamento sistemático.

\section{The use of the Khan Academy platform for mathematics teaching in Brazil: a systematic mapping}

Abstract. The insertion of digital technologies in education allows the teacher to adopt new pedagogical possibilities that aim to minimize the difficulties faced by students in subjects such as mathematics. In this context, the Khan Academy platform offers students a more active, dynamic and personalized learning, while allowing the teacher to monitor the performance of their classes and of each individual student. In this work, we present the results of a systematic mapping of literature that aimed to identify the works published in the last five years that use the Khan Academy platform to teach mathematics in Brazil. The results indicate that the Khan Academy platform has been gradually inserted in the process of teaching and learning mathematics at all levels of education in Brazil.

Key words: mathematics teaching, Khan Academy platform, systematic mapping.

\section{Introdução}

Em situações cotidianas observa-se que os estudantes brasileiros apresentam dificuldades com conceitos básicos de matemática, as quais são oriundas de deficiências na Educação Básica. Avaliações nacionais e internacionais revelam que a maioria dos estudantes brasileiros não possuem um conjunto de habilidades necessárias para aplicação de conhecimentos matemáticos no seu dia a dia. A Organização para a Cooperação e Desenvolvimento Econômico (OCDE) destaca a importância da capacidade de formular, empregar e interpretar a matemática significativamente e contextualmente, a partir da capacidade de descrever, explicar e prever fenômenos. Os resultados do Programa Internacional de Avaliação de Estudantes (PISA), realizado em 2018, corroboram para essa constatação. Os dados mostram que 68,1\% dos estudantes brasileiros não apresentaram o nível básico de matemática para o exercício pleno da 
cidadania (Brasil, 2019). Este cenário se repete nos resultados apresentados pelo Sistema de Avaliação da Educação Básica (Saeb), aplicado em 2019. Apesar de uma melhoria considerável nos índices em relação às edições anteriores do Saeb, os dados dessa última avaliação apontam que a maioria dos estudantes brasileiros do $3^{\circ}$ ano do Ensino Médio não possui o nível de qualidade esperado em matemática.

Visando minimizar as dificuldades enfrentadas pelos estudantes no processo de ensino e aprendizagem da matemática, vários estudos têm sido realizados com o propósito de sugerir a adoção de novas práticas pedagógicas (Meireles e Schimiguel, 2019). Uma das abordagens propostas consiste na inserção das tecnologias digitais na educação, especialmente no uso do computador e da Internet. Para Silveira et. al. (2019, p. 3), "utilizar tecnologias digitais para ensinar incita a criatividade e a interação do estudante, o que contribui para a compreensão dos conceitos em diferentes áreas do conhecimento". No ensino de matemática, o uso das tecnologias digitais provoca novas possibilidades pedagógicas, despertando o interesse dos alunos na construção do conhecimento matemático e no desenvolvimento de estruturas cognitivas (Kampff et. al., 2004).

Nesse contexto, a plataforma educacional Khan Academy vem se destacando por utilizar ferramentas multimídias que permitem o acesso online gratuito de materiais no formato de videoaulas, artigos, exercícios interativos e testes. Criada em 2006 pelo educador americano Salman Khan, a plataforma Khan Academy disponibiliza cursos em diversas áreas do conhecimento, tais como: matemática, biologia, química, física, astronomia, computação, entre outras, contemplando conteúdos desde a Educação Básica até o Ensino Superior. A plataforma oferece aos estudantes a possibilidade de um aprendizado mais ativo, dinâmico e personalizado (Tomazi, 2017). Conforme Hannel (2017), o ensino personalizado permite ao aluno autonomia no desenvolvimento de suas habilidades, estimulando o conhecimento e aguçando a curiosidade. Já aos professores, a plataforma Khan Academy pode ser vista como um recurso pedagógico que os auxilia no acompanhamento do desempenho de seus alunos, permitindo identificar as dificuldades no aprendizado de uma turma e/ou de cada aluno individualmente.

Nos últimos anos, algumas pesquisas nacionais vêm surgindo na literatura visando identificar as potencialidades associadas ao uso da plataforma Khan Academy no ensino de matemática. Nessa perspectiva, o objetivo geral deste trabalho é analisar como a plataforma Khan Academy vem sendo adotada no Brasil para o ensino de matemática da Educação Básica à Superior. Dessa forma, apresentamos um mapeamento sistemático da literatura com o objetivo de apresentar uma revisão ampla de estudos primários existentes nos últimos cinco anos.

\section{Mapeamento Sistemático da Literatura}

Para o desenvolvimento do mapeamento sistemático, adotamos o processo sugerido por Kitchenham e Charters (2007), que sugerem um processo dividido em três etapas distintas, que são: planejamento, condução e publicação dos resultados.

\subsection{Planejamento}

Iniciamos a etapa de planejamento identificando os trabalhos concernentes ao tema desta pesquisa: Santos e Gomes (2019) e Pinto et. al. (2019). Santos e Gomes (2019) apresentam uma revisão sistemática da literatura com o intuito de analisar como a plataforma Khan Academy está sendo utilizada para avanços da educação matemática, considerando trabalhos nacionais e internacionais. Os resultados revelam que a adoção 
da plataforma Khan Academy para o ensino de matemática se apresenta como uma alternativa viável e eficaz. Por sua vez, Pinto et. al. (2019) apresentam um levantamento bibliográfico com o objetivo de refletir sobre as experiências e práticas referentes à adoção da plataforma Khan Academy. De acordo com os autores, a inserção da plataforma Khan Academy no Brasil está subordinada à novidade e não necessariamente à inovação, com a necessidade da realização de estudos sobre o uso das tecnologias digitais na educação.

Com o intuito de obter uma visão mais abrangente do objetivo geral deste trabalho, foram levantadas as seguintes questões de pesquisa acerca do uso da plataforma Khan Academy para o ensino de matemática no Brasil:

- QP1: Em quais níveis a plataforma está sendo adotada?

- QP2: Quais estratégias de ensino estão sendo empregadas?

- QP3: Quais conteúdos matemáticos são explorados?

- QP4: Quais estratégias de investigação estão sendo empregadas para avaliação dos resultados?

Posteriormente, selecionamos as bases de dados a serem utilizadas na pesquisa. Optamos pela busca de trabalhos publicados em eventos e periódicos nacionais nas áreas de Educação, Educação Matemática e Informática na Educação. Além dessas bases de dados, também consideramos os periódicos da Coordenação de Aperfeiçoamento de Pessoal de Nível Superior (CAPES). Foram considerados na busca os trabalhos publicados nos últimos cinco anos (2015 - 2019), como também os periódicos publicados até o momento da pesquisa, realizada em repositórios online nos dias 7 e 8 de junho de 2020. A chave de busca escolhida ("khan academy" AND "matemática") visou abranger o maior número de trabalhos publicados.

Por fim, foram definidos alguns critérios de inclusão e exclusão. Como critério de inclusão, consideramos os trabalhos que apresentam como proposta uma avaliação acerca do uso da plataforma Khan Academy para o ensino de matemática na Educação Básica ou no Ensino Superior. Já os critérios de exclusão foram os seguintes:

- CE1: Trabalhos que não adotam a plataforma Khan Academy para o ensino de matemática;

- CE2: Trabalhos que não foram desenvolvidos no Brasil;

- CE3: Trabalhos que não apresentam uma avaliação sobre o uso da plataforma Khan Academy para o ensino de matemática;

- CE4: Trabalhos que apresentam revisões da literatura;

- CE5: Trabalhos indisponíveis para consulta.

\subsection{Condução}

$\mathrm{Na}$ etapa de condução, primeiramente realizamos a busca por trabalhos com base na chave de busca. Essa atividade resultou na identificação de 27 trabalhos. A Tabela 1 lista os eventos e periódicos analisados, além das respectivas quantidades de trabalhos publicados em cada ano. 
Tabela 1 - Resultado da busca nas bases de dados consideradas na pesquisa.

\begin{tabular}{|c|c|c|c|c|c|c|c|}
\hline Evento ou periódico & 每 & $\stackrel{0}{\overrightarrow{0}}$ & $\overline{\bar{N}}$ & $\stackrel{\infty}{\stackrel{\infty}{\tilde{N}}}$ & $\stackrel{\vec{\nabla}}{\vec{\nu}}$ & ণ્ণ & $\stackrel{\tilde{\pi}}{0}$ \\
\hline Congresso Nacional de Educação (CONEDU) & 0 & 0 & 0 & 0 & 0 & - & $\mathbf{0}$ \\
\hline Encontro de Paraibano de Educação Matemática (EPBEM) & - & 0 & - & 0 & - & - & $\mathbf{0}$ \\
\hline Encontro Nacional de Educação Matemática (ENEM) & - & 1 & - & - & 4 & - & 5 \\
\hline Congresso Internacional de Ensino de Matemática (CIEM) & - & - & 1 & - & - & - & 1 \\
\hline Simpósio Brasileiro de Informática na Educação (SBIE) & 0 & 0 & 0 & 0 & 0 & - & $\mathbf{0}$ \\
\hline Workshop de Informática na Escola (WIE) & 0 & 0 & 1 & 1 & 2 & - & 4 \\
\hline $\begin{array}{l}\text { Workshops do Congresso Brasileiro de Informática na } \\
\text { Educação }\end{array}$ & 0 & 0 & 0 & 0 & 1 & - & 1 \\
\hline $\begin{array}{l}\text { Congresso Internacional de Educação e Tecnologias e } \\
\text { Encontro de Pesquisadores em Educação à Distância (CIET- } \\
\text { ENPED) }{ }^{1}\end{array}$ & - & 1 & - & 2 & - & - & 3 \\
\hline Revista de Educação Matemática (Zetetike) & 0 & 0 & 0 & 0 & 0 & 0 & $\mathbf{0}$ \\
\hline Revista Eletrônica de Educação Matemática (REVEMAT) & - & - & - & - & - & 1 & 1 \\
\hline Revista Novas Tecnologias na Educação (RENOTE) & 1 & 0 & 0 & 0 & 0 & 0 & 1 \\
\hline Informática na Educação: Teoria \& Prática & 0 & 0 & 0 & 0 & 0 & 0 & $\mathbf{0}$ \\
\hline Periódicos da CAPES & 1 & 2 & 3 & 4 & 1 & 0 & 11 \\
\hline \multicolumn{7}{|c|}{ Total de trabalhos identificados } & 27 \\
\hline
\end{tabular}

Fonte: Autoria própria.

Após a busca dos trabalhos nas bases de dados, realizamos uma leitura completa dos mesmos e executamos a triagem dos que consideramos mais relevantes para nossa pesquisa, tomando como referência os critérios de inclusão e exclusão definidos na etapa de planejamento. Após esse passo, foram selecionados os dez trabalhos que são listados na Tabela 2. A Tabela 3 é semelhante à Tabela 1, considerando apenas os trabalhos selecionados.

Tabela 2 - Relação dos trabalhos classificados.

\begin{tabular}{|c|l|}
\hline Id & Referência \\
\hline T01 & Guimarães (2016) \\
\hline T02 & Santos, Santana e Santos (2016) \\
\hline T03 & Calazans (2017) \\
\hline T04 & Oliveira e Lima (2017) \\
\hline T05 & Souza e Giraffa (2017) \\
\hline T06 & Oliveira, Melo e Silva Júnior (2018) \\
\hline T07 & Vergana, Hinz e Lopes (2018) \\
\hline T08 & Almeida e Marinho (2019) \\
\hline T09 & Azevedo et. al. (2019) \\
\hline T10 & Araújo, Molina e Nantes (2020) \\
\hline
\end{tabular}

Fonte: Autoria própria.

\footnotetext{
${ }^{1}$ Antigo Simpósio Internacional de Educação à Distância e Encontro de Pesquisadores em Educação à Distância (SIED-ENPED).
} 
Tabela 3 - Resultado da busca nas bases de dados consideradas na pesquisa levando em conta apenas a lista dos trabalhos classificados a partir dos critérios de inclusão e exclusão.

\begin{tabular}{|c|c|c|c|c|c|c|c|}
\hline Evento ou periódico & 赵 & 룽 & 중 & $\stackrel{\infty}{\stackrel{\sim}{\vec{\nu}}}$ & 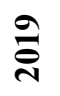 & હ્ત્తి & $\stackrel{\bar{\sigma}}{0}$ \\
\hline Encontro Nacional de Educação Matemática (ENEM) & - & 1 & - & - & 1 & - & 2 \\
\hline Congresso Internacional de Ensino de Matemática (CIEM) & - & - & 1 & - & - & - & 1 \\
\hline Workshop de Informática na Escola (WIE) & - & - & 0 & 1 & 1 & - & 2 \\
\hline $\begin{array}{l}\text { Workshops do Congresso Brasileiro de Informática na } \\
\text { Educação }\end{array}$ & - & - & - & - & 0 & - & $\mathbf{0}$ \\
\hline $\begin{array}{l}\text { Congresso Internacional de Educação e Tecnologias e } \\
\text { Encontro de Pesquisadores em Educação à Distância (CIET- } \\
\text { ENPED) }\end{array}$ & - & 1 & - & 0 & - & - & 1 \\
\hline Revista Eletrônica de Educação Matemática (REVEMAT) & - & - & - & - & - & 1 & 1 \\
\hline Revista Novas Tecnologias na Educação (RENOTE) & 0 & - & - & - & - & - & $\mathbf{0}$ \\
\hline Periódicos da CAPES & 0 & 0 & 2 & 1 & 0 & - & 3 \\
\hline \multicolumn{7}{|c|}{ Total de trabalhos selecionados } & 10 \\
\hline
\end{tabular}

Fonte: Autoria própria.

Em seguida, os trabalhos selecionados foram analisados visando à extração e mapeamento de dados correspondentes às questões de pesquisa. Os resultados serão apresentados na próxima seção ${ }^{2}$.

\section{Resultados e Discussão}

De acordo com os dados apresentados na Tabela 3, observamos que a maioria dos trabalhos foi publicada em eventos nacionais (seis artigos), sendo três na área de Educação Matemática (T03, T06 e T10) e três em Informática na Educação (T02, T04 e T09). Os demais trabalhos foram publicados em periódicos (T01, T05, T07 e T08).

Ao considerarmos os estados dos autores, verificamos que as regiões Sul e Nordeste concentram a maioria das publicações, sendo cinco trabalhos publicados oriundos da região Sul (T01, T05, T06, T07 e T08) e quatro da região Nordeste (T02, T04, T09 e T10). Esses trabalhos estão concentrados nos estados do Rio Grande do Sul (T05, T06, T07 e T08) e Rio Grande do Norte (T02 e T04). Os demais estados dessas regiões foram: Paraná (T01), Ceará (T09) e Bahia (T10), cada um com um trabalho publicado. A região Sudeste teve um trabalho publicado (T03), do Rio de Janeiro. Não encontramos nenhum trabalho com autores das regiões Norte e Centro-Oeste.

Levando-se em consideração o ano de publicação, conforme ilustra a Figura 1, vemos que o ano de 2017 concentra o maior número de publicações (T06, T07 e T08). Já nos anos de 2016 (T09 e T10), 2018 (T04 e T05) e 2019 (T02 e T03), foram publicados dois trabalhos por ano. Em 2020, foi encontrado um trabalho publicado até o momento da busca (T01).

Quanto ao tipo de pesquisa realizada, todos os trabalhos se enquadram como artigo de experiência. De acordo com Wieringa et al. (2006) citada por Falbo (2018, p. 19), um artigo de experiência "refere-se a trabalhos que discutem como alguém fez algo na prática (sem estar aplicando uma proposta de solução claramente colocada)".

\footnotetext{
${ }^{2}$ Uma sumarização dos resultados dos trabalhos selecionados pode ser acessada aqui.
} 
Figura 1 - Evolução do número de trabalhos publicados entre os anos de 2015 e 2020.

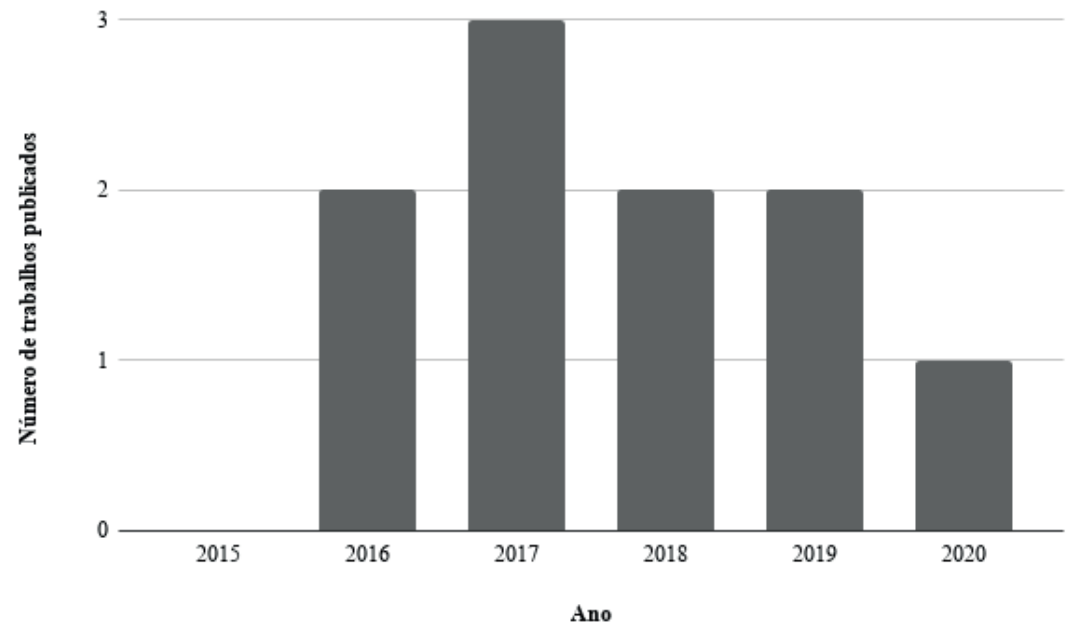

Fonte: Autoria própria.

$\mathrm{Na}$ sequência, apresentamos os dados coletados e detalhamos cada uma das questões de pesquisa.

\section{QP1: Em quais níveis a plataforma está sendo adotada?}

Conforme ilustra a Tabela 4, a maioria das pesquisas brasileiras está concentrada na Educação Básica (seis trabalhos), com três artigos abrangendo cada uma das seguintes etapas: Ensino Fundamental e Ensino Médio.

Os demais trabalhos foram desenvolvidos no âmbito do ensino superior, com $75 \%$ das pesquisas voltadas para alunos de cursos na área de exatas.

Tabela 4 - Mapeamento dos dados referentes à QP1.

\begin{tabular}{|c|l|l|}
\hline Id & Nível de ensino & Ano ou Curso \\
\hline T01 & Educação Básica (Ensino Médio) & Módulo I do Projeto e-Jovem \\
\hline T02 & Educação Básica (Ensino Médio) & $1^{\circ}$ ano \\
\hline T03 & Ensino Superior & Bacharelado Interdisciplinar em Ciências \\
\hline T04 & Educação Básica (Ensino Médio) & $2^{\circ}$ ano \\
\hline T05 & Ensino Superior & Licenciatura em Pedagogia \\
\hline T06 & Ensino Superior & Bacharelado em Tecnologia da Informação \\
\hline T07 & Educação Básica ( Ensino Fundamental) & $8^{\circ}$ ano \\
\hline T08 & Educação Básica ( Ensino Fundamental) & $8^{\circ}$ ano \\
\hline T09 & Ensino Superior & Bacharelado em Tecnologia da Informação \\
\hline T10 & Educação Básica ( Ensino Fundamental) & $6^{\circ}$ ano \\
\hline
\end{tabular}

Fonte: Autoria própria.

\section{QP2: Quais estratégias de ensino estão sendo empregadas?}

Quanto às estratégias de ensino adotadas, mapeadas na Tabela 5, observamos que $70 \%$ das pesquisas utilizaram a metodologia de ensino híbrido. Essa metodologia visa à combinação entre o aprendizado online (virtual) com o offline (presencial). As pesquisas que seguem essa metodologia apresentam a utilização da plataforma Khan Academy com o objetivo de fixação dos conteúdos matemáticos vistos em sala de aula por meio da recomendação de exercícios, vídeo aulas e artigos. 
Tabela 5 - Mapeamento dos dados referentes à QP2.

\begin{tabular}{|l|l|}
\hline Estratégia de ensino & Trabalhos \\
\hline Curso online à distância & T01; T02 \\
\hline Ensino híbrido & $\mathrm{T} 03 ; \mathrm{T} 04 ; \mathrm{T} 06 ; \mathrm{T} 07 ; \mathrm{T} 08 ; \mathrm{T} 09 ; \mathrm{T} 10$ \\
\hline Curso online presencial & $\mathrm{T} 05$ \\
\hline
\end{tabular}

Fonte: Autoria própria.

Outras estratégias identificadas adotam a plataforma Khan Academy para a realização de cursos online. Em dois dos dez trabalhos selecionados, os cursos ocorreram fora do ambiente escolar (educação à distância) e apenas um trabalho descreve uma experiência que ocorreu dentro da escola, com acompanhamento do professor (presencial). Nesses trabalhos a Khan Academy é utilizada com o propósito de oferecer cursos de nivelamento, visando à revisão de conteúdos de matemática básica.

\section{QP3: Quais conteúdos matemáticos são explorados?}

Com relação aos conteúdos matemáticos explorados na plataforma Khan Academy, nosso estudo aponta que 50\% dos trabalhos exploraram assuntos de matemática básica, conforme apresenta a Tabela 6 . Nos outros $50 \%$, os autores não especificam quais conteúdos foram abordados.

No T01, a autora menciona que os conteúdos selecionados em matemática básica na Khan Academy visam desenvolver o raciocínio lógico dos alunos em sala de aula. Nos demais trabalhos, fica evidente que os conteúdos trabalhados estão relacionados com os assuntos que estão sendo vistos em sala de aula.

Tabela 6 - Mapeamento dos dados referentes à QP3.

\begin{tabular}{|l|l|}
\hline Conteúdos & Trabalhos \\
\hline Álgebra & T02 e T03 \\
\hline Geometria espacial & T04 \\
\hline Frações & T05 e T10 \\
\hline Não específica & T01, T06, T07, T08 e T09 \\
\hline
\end{tabular}

Fonte: Autoria própria.

\section{QP4: Quais estratégias de investigação estão sendo empregadas para avaliação dos} resultados?

Verificamos que $60 \%$ dos trabalhos selecionados adotaram como estratégia de investigação a aplicação de métodos quantitativos para avaliação do uso da plataforma (T01, T02, T03, T04, T06 e T10). Já nos trabalhos T05, T07 e T09, métodos mistos foram utilizados para avaliar os estudos desenvolvidos. Apenas no trabalho T08, uma avaliação qualitativa foi conduzida.

De acordo com a Tabela 7, podemos notar que a maioria dos trabalhos que fazem uso de métodos quantitativos utiliza os resultados de avaliações tradicionais para mensurar o desempenho dos alunos que fazem uso da Khan Academy. Além das avaliações tradicionais, os trabalhos T02, T04 e T06 consideram também os relatórios gerados automaticamente pela plataforma Khan Academy. Os demais trabalhos fizeram uso de questionários e entrevistas.

Tabela 7 - Mapeamento dos dados referentes à QP4.

\begin{tabular}{|l|l|}
\hline Coleta de dados & Trabalhos \\
\hline Questionários & T07 \\
\hline Entrevistas & T05 \\
\hline Relatórios gerados pela plataforma & T02, T03, T04, T06 e T08 \\
\hline Avaliações tradicionais & T01, T02, T04, T06, T09 e T10 \\
\hline
\end{tabular}

Fonte: Autoria própria. 


\section{Ameaças à Validade}

O presente trabalho apresenta algumas ameaças à validade que podem ter comprometido os resultados apresentados. Uma delas diz respeito a limitações dos motores de busca utilizados para pesquisa em anais de eventos e periódicos. Assim, o conjunto de trabalhos selecionados pode não representar efetivamente todos os trabalhos publicados em língua portuguesa. Uma outra ameaça à validade está relacionada aos componentes humanos responsáveis pela avaliação dos trabalhos (extração e classificação dos dados).

\section{Conclusões}

O uso de tecnologias digitais no ensino de matemática já vem sendo amplamente discutido na literatura há alguns anos. No entanto, pesquisas visando à investigação dos benefícios atrelados à adoção da plataforma Khan Academy no processo de ensino e aprendizagem da matemática ainda são recentes. Nessa perspectiva, este trabalho apresentou um mapeamento sistemático da literatura com o propósito de identificar como essa plataforma está sendo utilizada e avaliada para o ensino de matemática por professores e pesquisadores brasileiros.

Observamos que a Educação Básica concentra a maioria dos estudos realizados no Brasil, sendo o ensino híbrido a principal metodologia de ensino adotada nas pesquisas apresentadas. Além disso, identificamos que a Khan Academy vem sendo amplamente utilizada para o ensino de conteúdos de matemática básica. Um dos motivos comumente apresentados para escolha dos conteúdos matemáticos a serem trabalhados está em minimizar as dificuldades dos alunos decorrentes da má formação adquirida na Educação Básica. Os relatórios de progresso dos alunos gerados automaticamente pela plataforma além das avaliações tradicionais são os métodos mais comuns de avaliação adotados pelos estudos desenvolvidos no Brasil.

Diante do exposto, é razoável inferir que, na perspectiva dos alunos e dos professores brasileiros, a adoção da Khan Academy como ferramenta de suporte pode ser benéfica para o processo de ensino e aprendizagem de conteúdos matemáticos. No entanto, os resultados do presente trabalho revelam que estudos acerca do uso da plataforma no ensino de matemática ainda vem sendo pouco explorado no Brasil nos últimos anos, principalmente para o ensino de conteúdos mais avançados de matemática no Ensino Superior. Nesse sentido, se faz necessário a realização de novas pesquisas visando suprir essa lacuna.

\section{Agradecimentos}

O presente trabalho foi realizado com apoio da $\mathrm{UEPB} / \mathrm{CNPq}$, através do Programa de Iniciação Científica da Universidade Estadual da Paraíba (PIBIC - cota 2020/2021).

\section{Referências}

ALMEIDA, L. F. A.; MARINHO, F. C. V. Khan Academy: Uma experiência de uso no processo de ensino e aprendizagem. In: Anais eletrônico do Encontro Nacional de Educação Matemática. 2019. Disponível em: $<$ https://www.sbemmatogrosso.com.br/eventos/index.php/enem/2019/paper/view/2960/ 1250>. Acesso em: 9 jun. 2020. 
ARAÚJO, V. S.; MOLINA, L. P. P.; NANTES, E. A. S. Khan Academy: uma possibilidade para as aulas de matemática. Revista Eletrônica de Educação Matemática, v. 15, n. 1, p. 1-19, 2020.

AZEVEDO, A. V. M.; MEDEIROS, L. L. T.; BEZERRA, V. H. C.; OLIVEIRA, A. I. S. Uso da Khan Academy como Avaliação Continuada em Matemática Elementar. In: Anais do Workshop de Informática na Escola, p. 1094, 2019.

BRASIL. Ministério da Educação. INEP. Relatório Brasil no PISA 2018, versão preliminar, Diretoria de Avaliação da Educação Básica (DAEB), Poder Executivo, Brasília, DF, 2019. Disponível em: <http://portal.inep.gov.br/web/guest/acoesinternacionais/pisa/resultados >. Acesso em: 20 mai. 2020.

CALAZANS, M. V. Khan Academy como recurso para o Ensino Híbrido: Relato de aplicação na Universidade Federal do Sul da Bahia. In: VII Congresso Internacional de Ensino de Matemática. 2017.

FALBO, R. A. Mapeamento Sistemático. Vitória: Universidade Federal do Espírito Santo. Disponível em: http://www.inf.ufes.br/ falbo/files/MP/TP/Sobre_MS.pdf. Acesso em: 08 jun. 2020.

GUIMARÃES, M. Khan Academy: Utilização no processo de construção da lógica à luz da inteligência matemática de Gardner. In: Anais do Simpósio Internacional de Educação a Distância e Encontro de Pesquisadores em Educação a Distância, 2016.

HANNEL, K. Um método e suas práticas pedagógicas para atingir a aprendizagem significativa. Tese (Doutorado em Informática na Educação) - Universidade Federal do Rio Grande do Sul. Porto Alegre, p. 134. 2017.

KAMPFF, A. J. C.; MACHADO, J. C.; CAVEDINI, P. Novas tecnologias e educação matemática. RENOTE-Revista Novas Tecnologias na Educação, v. 2, n. 2, 2004.

KITCHENHAM, B.A., CHARTERS, S., Guidelines for performing systematic literature reviews in software engineering. Tech. Rep. EBSE-2007-01, Keele University, 2007.

MEIRELES, S. M.; SCHIMIGUEL, J. Tendências de tecnologia para o ensino de matemática. UNIÓN-REVISTA IBEROAMERICANA DE EDUCACIÓN MATEMÁTICA, v. 15, n. 57, p. 95-106, 2019.

OLIVEIRA, A. I.; MELO, E.; SILVA JÚNIOR, J. Uso do Khan Academy como Avaliação Continuada em Cálculo I. In: Anais do Workshop de Informática na Escola, p. 373. 2018.

OLIVEIRA, H. S.; LIMA, M. F. W. P. Utilização da Plataforma Khan Academy na Resolução de Exercícios de Matemática. Scientia cum Industria, v. 5, n. 2, p. 66-72, 2017.

PINTO, J. C.; LÚCIA, S. F.; MANGELA, M. F. Plataforma Khan Academy: Um levantamento bibliográfico para além da novidade educacional. In: Anais do XIII ENEM - Encontro Nacional de Educação Matemática. Cuiabá, MT, Brasil: 2019.

SANTOS, P. V. M.; SANTANA, A. S. A.; SANTOS, T. J. P. Khan Academy: Uma proposta de nivelamento em matemática no IFBA Campus Simões Filho. In: Anais eletrônico do Encontro Nacional de Educação Matemática. 2016. Disponível em: $<$ http://www.sbembrasil.org.br/enem2016/anais/pdf/8303_4245_ID.pdf $>$. Acesso em: 9 jun. 2020 . 
SANTOS, Y.; GOMES, A. Análise da utilização da plataforma Khan Academy para a educação matemática. In: Anais do XXV Workshop de Informática na Escola. Brasília, DF, Brasil: 2019.

SILVEIRA, D. S.; FONSECA, D. A.; LAURINO, D. P. O dar-se conta da formação de professores de matemática no operar de tecnologias digitais no ensino superior. Brazilian Journal of Development, v. 5, n. 8, 2019.

SOUZA, C. T.; GIRAFFA, L. M. M. O uso da plataforma Khan Academy como suporte à formação inicial de professores dos anos iniciais. In: Anais IV Seminário Internacional Pessoa Adulta, Saúde e Educação (SIPASE), 2017.

TOMAZI, D. R. A plataforma Khan Academy para o ensino de matemática do $4^{\circ}$ ano do ensino fundamental: aspectos teóricos e práticos. 2017.

VERGARA, A. C. E.; HINZ, V. T.; LOPES, J. L. B. Como Significar a Aprendizagem de Matemática Utilizando os Modelos de Ensino Híbrido. Revista Thema, v. 15, n. 3, p. 885-904, 2018.

WIERINGA, R., MAIDEN, N. A. M., MEAD, N. R. \& ROLLAND, C., Requirements Engineering Paper Classification and Evaluation Criteria: a Proposal and a Discussion. Requirements Engineering, 11(1), 102-107. 2006. 